\title{
Comparison of Duke Activity Status Index with cardiopulmonary exercise testing in cancer patients
}

\author{
Michael H.-G. Li ${ }^{1} \cdot$ Vladimir Bolshinsky² $\cdot$ Hilmy Ismail ${ }^{1} \cdot$ Kwok-Ming Ho $^{3} \cdot$ Alexander Heriot $^{2,4} \cdot$ Bernhard Riedel $^{1,4}$
}

Received: 12 February 2018 / Accepted: 23 May 2018 / Published online: 29 May 2018

(c) Japanese Society of Anesthesiologists 2018

\begin{abstract}
Purpose The Duke Activity Status Index (DASI), a patient-administered questionnaire, is used to quantify functional capacity in patients undergoing cancer surgery.

Methods This retrospective cohort study assessed whether the DASI was accurate in predicting peak oxygen consumption $\left(\mathrm{pVO}_{2}\right)$ that was objectively measured using cardiopulmonary exercise testing (CPET) in 43 consecutive patients scheduled for elective major cancer surgery at a tertiary cancer centre. The primary outcome measured the limits of agreement between DASI-predicted $\mathrm{pVO}_{2}$ and actual measured $\mathrm{pVO}_{2}$.

Results The study population was elderly (median 63 years, interquartile range 18), 58\% were male, with the majority having intraabdominal cancer surgery. Although the DASI scores were statistically related to the measured $\mathrm{pVO}_{2}(N=43$, adjusted $\left.R^{2}=0.20, p=0.002\right)$, both the bias $\left(8 \mathrm{ml} \mathrm{kg}^{-1} \mathrm{~min}^{-1}\right)$ and $95 \%$ limits of agreement $\left(19.5\right.$ to $\left.-3.4 \mathrm{ml} \mathrm{kg}^{-1} \mathrm{~min}^{-1}\right)$ between the predicted and measured $\mathrm{pVO}_{2}$ were large. Using some of the individual components, recalibrating the intercept and regression coefficient of the total DASI score did not substantially improve its ability to predict the measured $\mathrm{pVO}_{2}$. Conclusion In summary, both the limits of agreement and bias between the measured and DASI-predicted $\mathrm{pVO}_{2}$ were substantial. The DASI-predicted $\mathrm{pVO}_{2}$ based on patient's assessment of their functional status could not be considered a reliable surrogate of measured $\mathrm{pVO}_{2}$ during CPET for the population of patients pending major cancer surgery and cannot, therefore, be used as a triage tool for referral to CPET centres for objective risk assessment.
\end{abstract}

Keywords Preoperative care · Cancer · Exercise test · Questionnaire · Maximal oxygen uptake · Anaerobic threshold · Lactate threshold

Electronic supplementary material The online version of this article (https://doi.org/10.1007/s00540-018-2516-6) contains supplementary material, which is available to authorized users.

Michael H.-G. Li

Michael.Huagen.Li@gmail.com

1 Department of Cancer Anaesthesia, Perioperative and Pain Medicine, Peter MacCallum Cancer Centre, Melbourne, Australia

2 Division of Cancer Surgery, Peter MacCallum Cancer Centre, Melbourne, Australia

3 Department of Intensive Care Medicine, School of Population Health \& School of Veterinary and Life Sciences, Royal Perth Hospital, University of Western Australia \& Murdoch University, Perth, Australia

4 Melbourne Medical School, University of Melbourne, Melbourne, Australia

\section{Introduction}

Elderly and frail patients scheduled to undergo major cancer surgeries are increasing. Impaired functional capacity from frailty, the natural effects of aging, sedentary lifestyle, and cancer biology are associated with increased postoperative complications, prolonged length of hospital stay, and increased risk of death after major cancer surgery [1]. Studies have also shown that neoadjuvant cancer therapy can cause a reduction in oxygen consumption $\left(\mathrm{VO}_{2}\right)$ at Anaerobic Threshold (AT) and at peak exercise $\left(\mathrm{pVO}_{2}\right)$ of up to $30 \%\left(2-3 \mathrm{ml} \mathrm{kg}^{-1} \mathrm{~min}^{-1}\right)$ when measured using serial cardiopulmonary exercise testing (CPET) [2-4].

CPET, currently considered the gold standard in assessing functional capacity, is a dynamic, symptom-limited, noninvasive test that provides objective analysis of the functional capacity of the integrated cardiovascular, pulmonary, haematinic, and cellular metabolic systems [5-7]. CPET is 
increasingly used in the perioperative setting for preoperative risk stratification and, more recently, to guide preoperative optimisation strategies $[8,9]$. The predominant CPETderived parameters have been shown to be strong predictors of adverse outcomes following major surgery and include AT, $\mathrm{pVO}_{2}$, and minute equivalents for $\mathrm{CO}_{2}$ (ventilation/ carbon dioxide production; $\mathrm{Ve} / \mathrm{VCO}_{2}$ ). A recent systematic review demonstrated that an $\mathrm{AT}<10.1$ and $<10.9 \mathrm{ml}$ $\mathrm{kg}^{-1} \mathrm{~min}^{-1}$ were strong predictors of increased morbidity and mortality, respectively, in patients undergoing intraabdominal surgery [10]. Similarly, $\mathrm{pVO}_{2}<15 \mathrm{ml} \mathrm{kg}^{-1} \mathrm{~min}^{-1}$ is also an independent predictor of postoperative morbidity and mortality $[11,12]$.

The promising role of CPET as a perioperative risk assessment tool, however, is offset by it being resourceintensive, requiring skilled personnel and specialized equipment [13]. The Duke Activity Status Index (DASI: range between 0 and 58), a static questionnaire that has been developed almost 3 decades ago as a simple, quick, and cost-free surrogate measure of $\mathrm{pVO}_{2}$, is, therefore, appealing to identify at-risk patients for triage to a CPET laboratory. Based on simple yes or no answers to 12 questions related to the patient's activities of daily living (Appendix 1 in supplementary material), $\mathrm{pVO}_{2}$ can be estimated using the following equation: $\mathrm{pVO}_{2}=$ DASI score $\times 0.43+9.6[14,15]$. Although it was originally developed to monitor clinical progress of cardiovascular patients, the DASI has also been shown to have a modest correlation with $\mathrm{pVO}_{2}$ measured by CPET in patients undergoing general intraabdominal surgery [16]. Specifically, question 4-the ability to climb a flight of stairs-is frequently used in anaesthetic practice to estimate metabolic equivalents.

Nevertheless, the validity of using DASI to predict the true functional capacity of major cancer surgical patients has not been specifically examined. Cancer surgical patients often have preoperative neoadjuvant chemoradiotherapy that may induce deconditioning and confound the ability of the DASI to predict $\mathrm{pVO}_{2}$. We hypothesised that the DASI-predicted $\mathrm{pVO}_{2}$ may not be a good predictor of the functional capacity of major cancer surgical patients. In this study, (1) we compared the bias and limits of agreement between the measured and DASI-predicted $\mathrm{pVO}_{2}$ in a cohort of major cancer surgical patients; and (2) if proved unsatisfactory, whether recalibration of the predictive coefficient of each domain (or question) of the DASI questionnaire would improve its predictive ability.

\section{Methods}

Following Peter MacCallum Cancer Centre Human Research Ethics Committee approval (16/136R-November 2016), we undertook a retrospective cohort study of 43 consecutive patients who were scheduled for major cancer surgery (defined as intracavity tumour surgery lasting for more than $2 \mathrm{~h}$ ), who, during their preoperative workup, were referred to our CPET service (January-June 2014), and had a concurrent DASI questionnaire administered. Referral of patients for CPET assessment was initiated by surgeons based on the standardised hospital-based CPET referral guidelines (Appendix 2 in supplementary material), with a predominant casemix of colorectal and upper gastrointestinal cancers. All patients who underwent CPET during this period were included. Patients who were unable to perform the test due to pain upon cycling and those who had neurological or severe cognitive deficits were excluded from CPET in the study centre. Cases with missing data were excluded from analysis. Data were collected in November 2016 through review of hospital electronic data and medical records. All cases were de-identified prior to statistical analysis.

CPET was performed as per the American Thoracic Society/American College of Chest Physicians (ATS/ACCP) practice guidelines [17]. A static respiratory function test was performed prior to exercise. Baseline data, collected with the patient at rest for $3 \mathrm{~min}$, included: blood pressure, continuous 12-lead electrocardiogram (ECG), oxygen saturation, and breath-by-breath gas exchange through a tightfitting mask (CardiO2/CP System, Medical Graphics Corporation, USA). During the exercise phase, patients cycled at 60-70 RPM on a cycle ergometer: 3 min unloaded cycling, then a ramp protocol of $20 \mathrm{~W} \mathrm{~min}{ }^{-1}$ through increasing pedal resistance until peak exercise, and then 5 min of unloaded cycling during the recovery period. A clinician was present throughout. Ramping was stopped after achieving peak exercise at the patient or clinician's discretion, either due to patient fatigue, dyspnoea, chest pain, leg pain, or signs of myocardial ischemia, hypotension, or arrhythmia. AT was determined using both the $V$-slope and ventilator equivalents [18] methods by two experienced anaesthetists trained in CPET. Two independent reviewers analysed and crosschecked the CPET data to ensure accuracy.

Data collected included patient demographics (age, gender, height, weight, etc.), history of prior administration of chemotherapy and/or radiotherapy within the last 6 months, objective measures of CPET-derived AT and $\mathrm{pVO}_{2}$ using gas-exchange analysis, DASI score, and the corresponding predicted $\mathrm{pVO}_{2}$. DASI was measured using a self-administered questionnaire [14] as part of routine preoperative assessment immediately before performance of CPET. The DASI was not available to the clinician interpreting the CPET results. Peak $\mathrm{VO}_{2}$, AT, age, height, and weight were evaluated as nominal values, whereas gender and prior chemoradiotherapy (yes or no) were described as categorical.

The primary outcome of this study evaluated the limits of agreement between DASI-predicted $\mathrm{pVO}_{2}$ and actual 
measured $\mathrm{pVO}_{2}$. Secondary outcomes investigated (a) whether the agreement between DASI-predicted $\mathrm{pVO}_{2}$ and actual measured $\mathrm{pVO}_{2}$ was affected by prior chemoradiotherapy; (b) the ability of the raw DASI score to predict (1) measured $\mathrm{pVO}_{2}$ and (2) anaerobic threshold; and (c) whether recalibrating the predictive coefficient associated with each domain (or question) of the DASI questionnaire would improve its predictive ability [19].

\section{Statistical analyses}

We assessed the bias and 95\% limits of agreement between the predicted and measured $\mathrm{pVO}_{2}$ using a Bland-Altman plot, stratified by whether the patients had received chemoradiotherapy within 6 months of CPET. A scatter plot was used to assess how the measured $\mathrm{pVO}_{2}$ and AT were related to the DASI scores and the DASI-derived-predicted $\mathrm{pVO}_{2}$. Acceptable limits of agreement was considered to be a $\mathrm{pVO}_{2}$ value of $3 \mathrm{ml} \mathrm{kg}^{-1} \mathrm{~min}^{-1}$ [20]. Normality of the response variables $\left(\mathrm{pVO}_{2}\right.$ and $\left.\mathrm{AT}\right)$ was assessed by $\mathrm{Q}-\mathrm{Q}$ plots and formally tested using the Shapiro-Wilk test-the most powerful statistical test for normality [21], before they were modelled using linear regression by the DASI score.

We then assessed whether recalibrating the intercept and predictive coefficient of the total DASI score or combining age, gender, and recent chemoradiotherapy with each individual DASI question's score would improve the ability of DASI's ability to predict the measured $\mathrm{pVO}_{2}$. In the latter analysis, a parsimony linear regression model was obtained by eliminating covariates in the model by optimising the adjusted R-square and the area under the receiver-operating characteristic (AUROC) curve of the model to discriminate between those patients with $\mathrm{pVO}_{2} \geq 15$ and $<15 \mathrm{ml} \mathrm{kg}^{-1}$ $\mathrm{min}^{-1}$. Finally, we also assessed the AUROC of the DASI score to discriminate between those patients with AT $\geq 11 \mathrm{ml}$ $\mathrm{kg}^{-1} \mathrm{~min}^{-1}$ and those with AT $<11 \mathrm{ml} \mathrm{kg}^{-1} \min ^{-1}$ (a cutpoint considered to be associated with increased perioperative surgical risk) [10, 11].

All statistical tests were performed using Predictive Analytics Software Statistics 22 (IBM Corporation, New York, 2013) and MedCalc (version 17.4 MedCalc Software bvba, Ostend, Belgium; 2017), and a $p$ value $<0.05$ was taken as statistically significant.

\section{Results}

A total of 45 cases were eligible for this study. Two cases were missing at least one data entry point in the DASI score and were excluded from further analyses. Most patients were elderly (median 63 years, interquartile range 18 years); male $(58 \%)$ patients scheduled for intraabdominal cancer surgery (with the exception of two cases having thoracic surgery) (Table 1).

The bias (or mean difference) between the predicted and measured $\mathrm{pVO}_{2}$ was large (on average the predicted overestimated measured $\mathrm{pVO}_{2}$ by $8 \mathrm{ml} \mathrm{kg} \mathrm{kg}^{-1} \min ^{-1}, 95 \%$ CI $6.2-9.8 ; p=0.0001$ ), and the $95 \%$ limits of agreement of the differences between the two measurements was wide (19.5 to $-3.4 \mathrm{ml} \mathrm{kg}^{-1} \mathrm{~min}^{-1}$ ). The predicted $\mathrm{pVO}_{2}$ tended to further overestimate the measured $\mathrm{pVO}_{2}$ as the measured $\mathrm{pVO}_{2}$ increased (Fig. 1). The bias and 95\% limits of agreement between the predicted and measured $\mathrm{pVO}_{2}$ appeared to be similar between patients who had recent chemoradiotherapy (mean bias $8.5,95 \%$ limits of agreement 20.5 to $-3.5 \mathrm{ml} \mathrm{kg}^{-1} \mathrm{~min}^{-1}$ ) and those who did not (mean bias $7.8,95 \%$ limits of agreement 19.1 to $-3.6 \mathrm{ml} \mathrm{kg}^{-1} \mathrm{~min}^{-1}$; Figs. 2, 3, respectively).

Normality of the response variables $\left(\mathrm{pVO}_{2}\right.$ and AT) was confirmed by Q-Q plots and the Shapiro-Wilk test $(p=0.190$ and $p=0.206$, respectively). DASI-predicted $\mathrm{pVO}_{2}$ was only weakly linearly associated with the actual $\mathrm{pVO}_{2}(N=43$, adjusted $R^{2}=0.20 ; p=0.002$; Fig. 4 ), and the DASI scores were not statistically significantly related to the AT $(p=0.111$; Fig. 5). The raw DASI scores had a modest ability to discriminate between those who had a $\mathrm{pVO}_{2}>15 \mathrm{ml}$ $\mathrm{kg}^{-1} \min ^{-1}$ and those who did not (AUROC $=0.77,95 \%$ CI 0.61-0.88), but it did not accurately discriminate between patients with a high $\left(>11 \mathrm{ml} \mathrm{kg}^{-1} \mathrm{~min}^{-1}\right)$ and low AT $\left(\leq 11 \mathrm{ml} \mathrm{kg}^{-1} \mathrm{~min}^{-1}\right)$ with AUROC $=0.53,95 \% \mathrm{CI}$ $0.33-0.73, p=0.791$; Figs. 6,7 , respectively.

Recalibrating the intercept and regression coefficient of the total DASI score (our revised predicted $\mathrm{pVO}_{2}$ with DASI score alone $=0.115 \times$ DASI score +13.3 ; adjusted $R^{2}=0.20$ and AUROC to predict $>15 \mathrm{ml} \mathrm{kg}^{-1} \mathrm{~min}^{-1}=0.72$, 95\% CI 0.54-0.90) also did not improve its ability to predict the measured $\mathrm{pVO}_{2}$ in a substantial fashion. Further permutations, by including gender, but not age, or recent chemoradiotherapy [revised predicted $\mathrm{VO}_{2}$ with DASI and

Table 1 Summary of patient characteristics and DASI-predicted and CPET measured functional capacity measures

\begin{tabular}{lc}
\hline Sex, male; $\mathrm{n}(\%)$ & $25(58.10 \%)$ \\
Age, years; median (IQR) & $63.00(18.00)$ \\
Body Mass Index, $\mathrm{kg} \mathrm{m}^{-2}$; median (IQR) & $25.92(8.07)$ \\
Chemoradiotherapy within preceding 6 months; $n(\%)$ & $15(34.88 \%)$ \\
DASI score; median (IQR) & $37.45(27.25)$ \\
DASI-predicted pVO $\mathrm{ml} \mathrm{kg}^{-1} \mathrm{~min}^{-1}[=0.43 \times$ DASI & $25.70(11.72)$ \\
$\quad$ score + 9.6]; median (IQR) & \\
CPET AT, $\mathrm{ml} \mathrm{kg}^{-1} \mathrm{~min}^{-1} ;$ median (IQR) & $11.8(3.42)$ \\
CPET peak $\mathrm{VO}_{2}, \mathrm{ml} \mathrm{kg}^{-1}$ min $^{-1}$; median (IQR) & $17.64(5.78)$ \\
\hline
\end{tabular}

$I Q R$ interquartile range, CPET cardiopulmonary exercise test, peak $\mathrm{VO}_{2}$ maximal oxygen uptake, $A T$ anaerobic threshold, DASIDuke Activity Status Index 
Fig. 1 Bland-Altman analysis of DASI-predicted and CPET measured oxygen consumption at peak exercise $\left(\mathrm{pVO}_{2}\right)$. This figure shows that the bias (or mean difference) between the predicted and measured $\mathrm{pVO}_{2}$ was large (on average the predicted $>$ measured $\mathrm{pVO}_{2}$ by $8 \mathrm{ml}$ $\mathrm{kg}^{-1} \min ^{-1}, 95 \%$ CI 6.2-9.8; $p=0.0001$ ), and the $95 \%$ limits of agreement of the differences between the two measurements were wide ( 19.5 to $-3.4 \mathrm{ml}$ $\left.\mathrm{kg}^{-1} \min ^{-1}\right)$
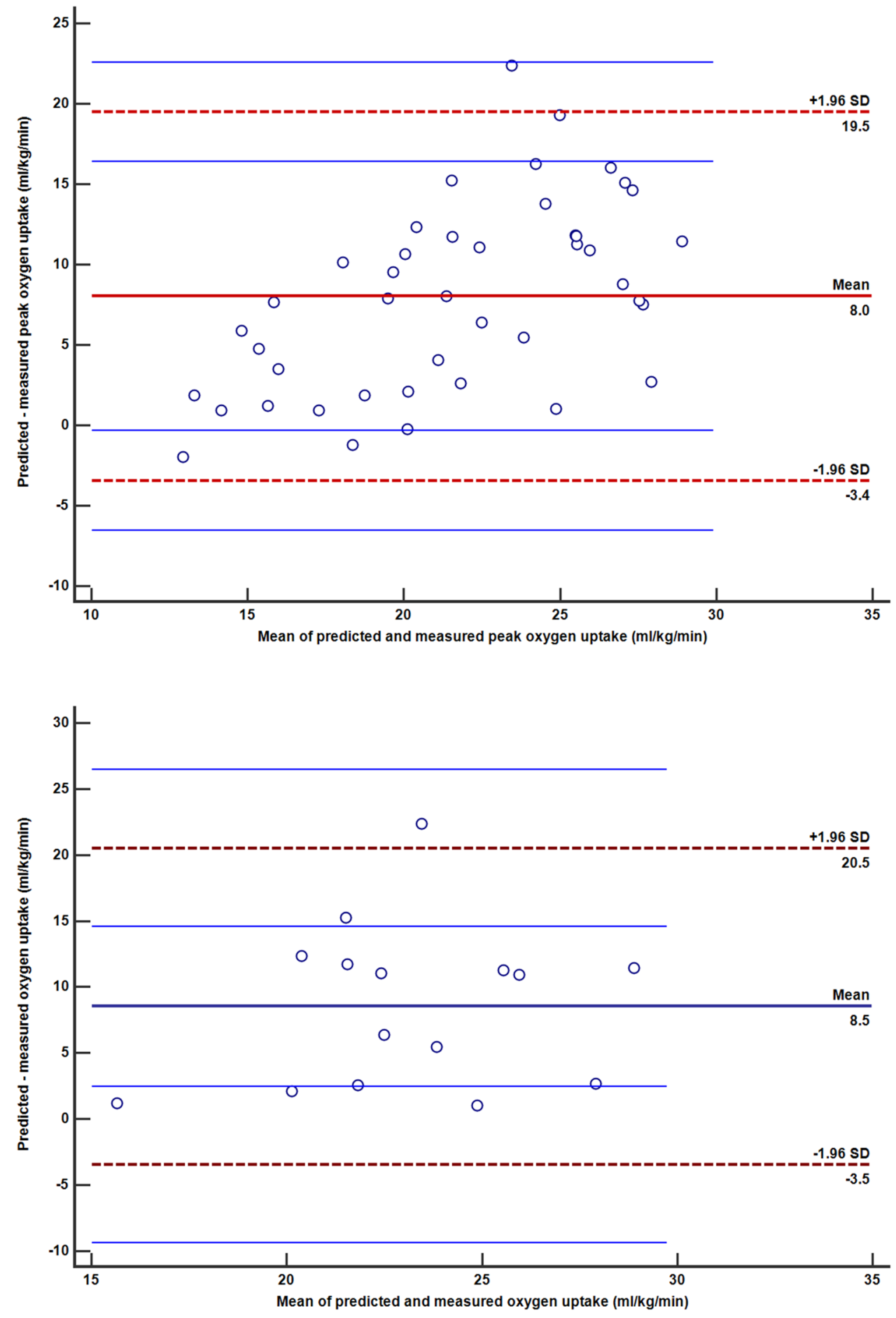

for those treated with chemoradiotherapy within 6 months
Fig. 2 Bland and Altman Plot between measured and predicted peak oxygen uptake for those who had recent chemoradiotherapy gender $=0.11 \times$ DASI score + male $\times(1.93)+12.4$; adjusted $R^{2}=0.25$ and AUROC to predict $>15 \mathrm{ml} \mathrm{kg}^{-1} \mathrm{~min}^{-1}=0.74$, 95\% CI 0.58-0.91], only slightly improved the revised DASI model to predict the measured $\mathrm{pVO}_{2}$.

Using individual scores for each of the DASI questions improved the ability to predict the measured $\mathrm{pVO}_{2}$ statistically (with a revised raw data model derived to predict $\mathrm{pVO}_{2}=10.294+$ male $\times 2.045+\mathrm{Q} 5 \times 0.203+\mathrm{Q} 6 \times$ $1.417+\mathrm{Q} 10 \times 0.574$; adjusted $R^{2}=0.37$ and AUROC to predict $>15 \mathrm{ml} \mathrm{kg}^{-1} \mathrm{~min}^{-1}=0.74,95 \%$ CI $0.59-0.89$; Fig. 8), but this, by no means, could be considered a reliable replacement of measuring $\mathrm{pVO}_{2}$ directly. 
Fig. 3 Bland and Altman Plot between measured and predicted peak oxygen uptake for those without recent chemoradiotherapy

Fig. 4 Association between Duke Activity Status Index and maximal oxygen uptake, and the straight line indicates the linear regression line
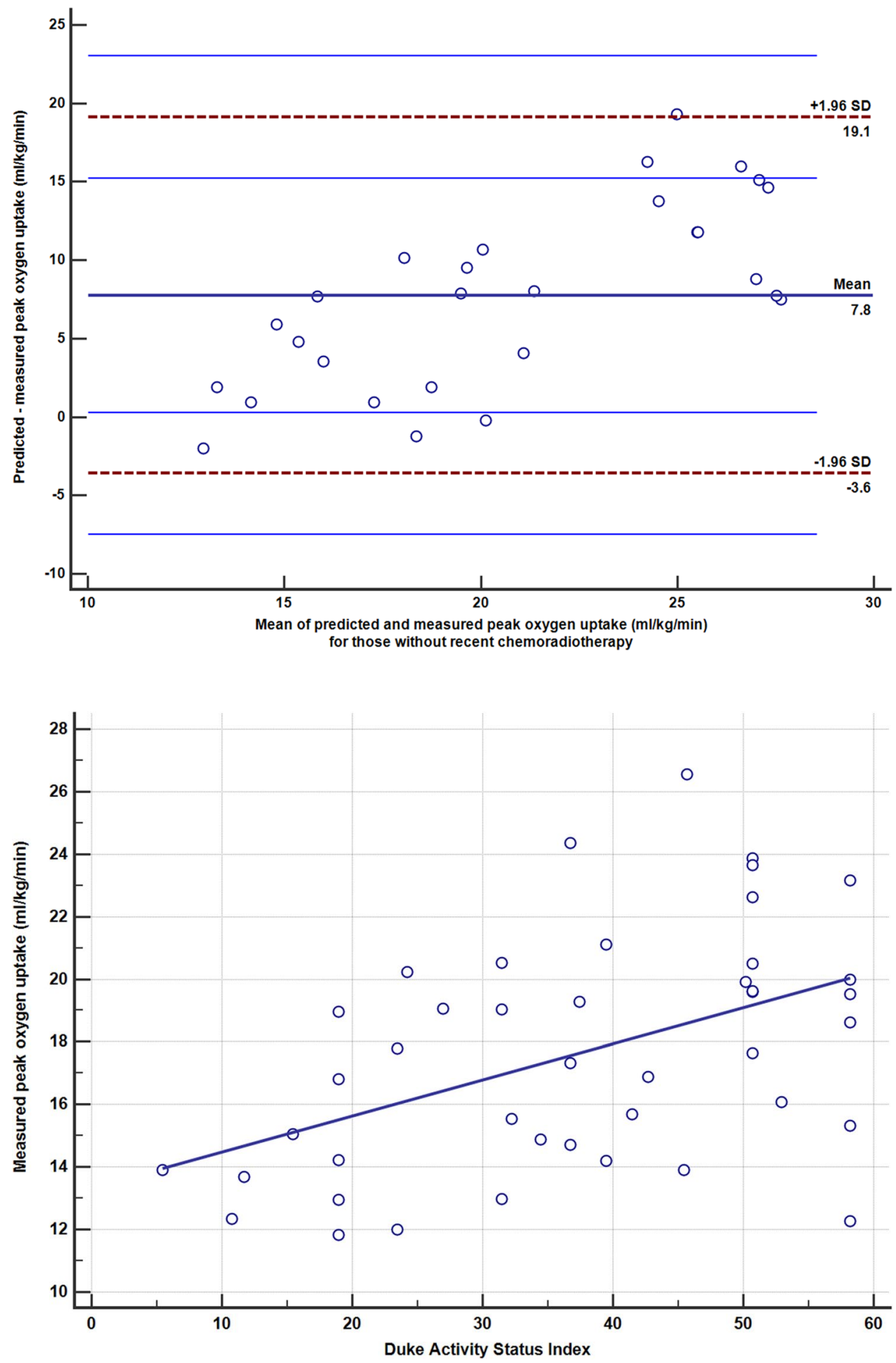

\section{Discussion}

\section{Comparison with the existing literature}

Our findings in the cancer surgery population demonstrated that DASI-predicted $\mathrm{pVO}_{2}$ had a substantial bias and wide $95 \%$ limits of agreement compared to the measured $\mathrm{pVO}_{2}$. This result was different from the previous reports on the general population $\left(R^{2}=0.64\right)$ and in the non-cancer specific patient population undergoing intraabdominal surgery $\left(R^{2}=0.45\right)[14,16]$. The intraabdominal cohort, investigated by Struthers et al., recorded only types of surgery and did not specify oncological status: vascular 
Fig. 5 Association between Duke Activity Status Index and anaerobic threshold, and the straight line indicates the linear regression line

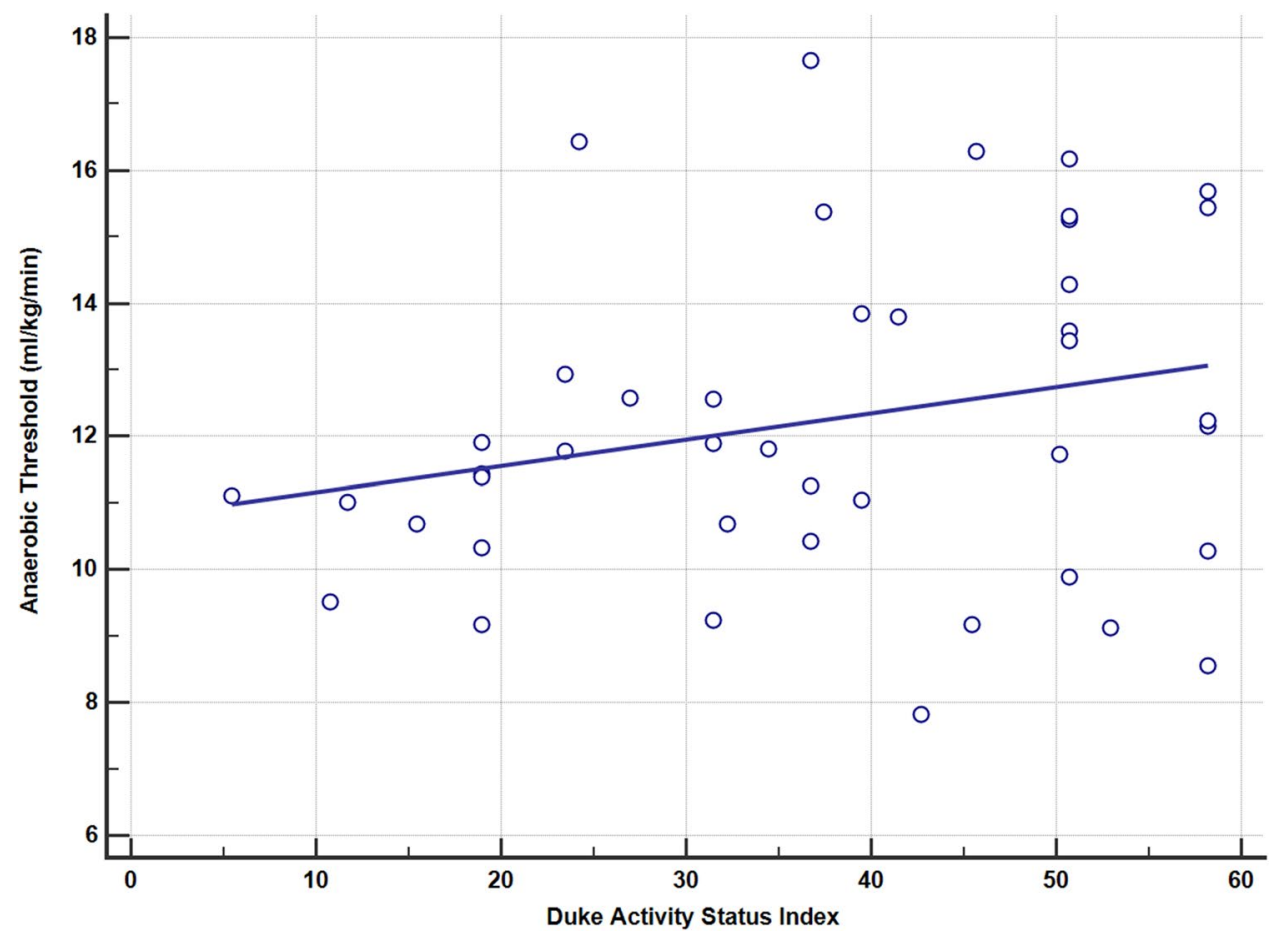

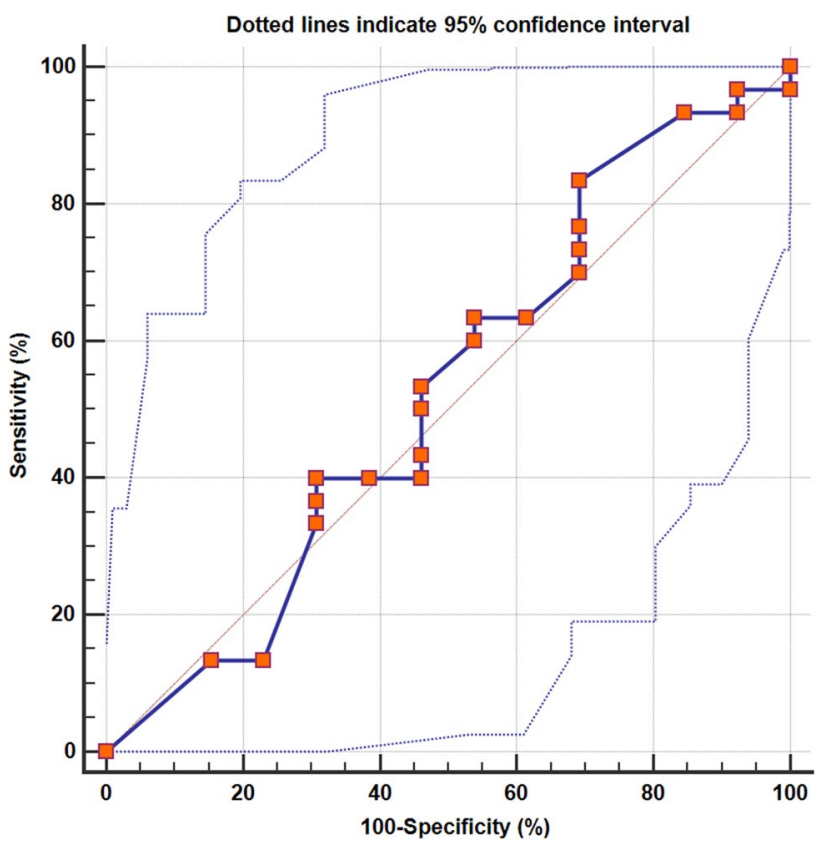

Fig. 7 Area under the receiver-operating characteristic curve for Duke Activity Status Index to differentiate an anaerobic threshold $<11$ against $\geq 11 \mathrm{ml} \mathrm{kg}^{-1} \min ^{-1}=0.526$ (95\% CI $0.326-0.725$, $p=0.791)$

to predict AT $>11 \mathrm{ml} \mathrm{kg}{ }^{-1} \mathrm{~min}^{-1}$ (AUC $0.53,95 \% \mathrm{CI}$ $0.33-0.73, p=0.791$ ) in our cancer surgical cohort. Our study confirms that the DASI reported in the development cohort [14] at best only offers a modest correlation with CPET results and is unsuitable for use in the 


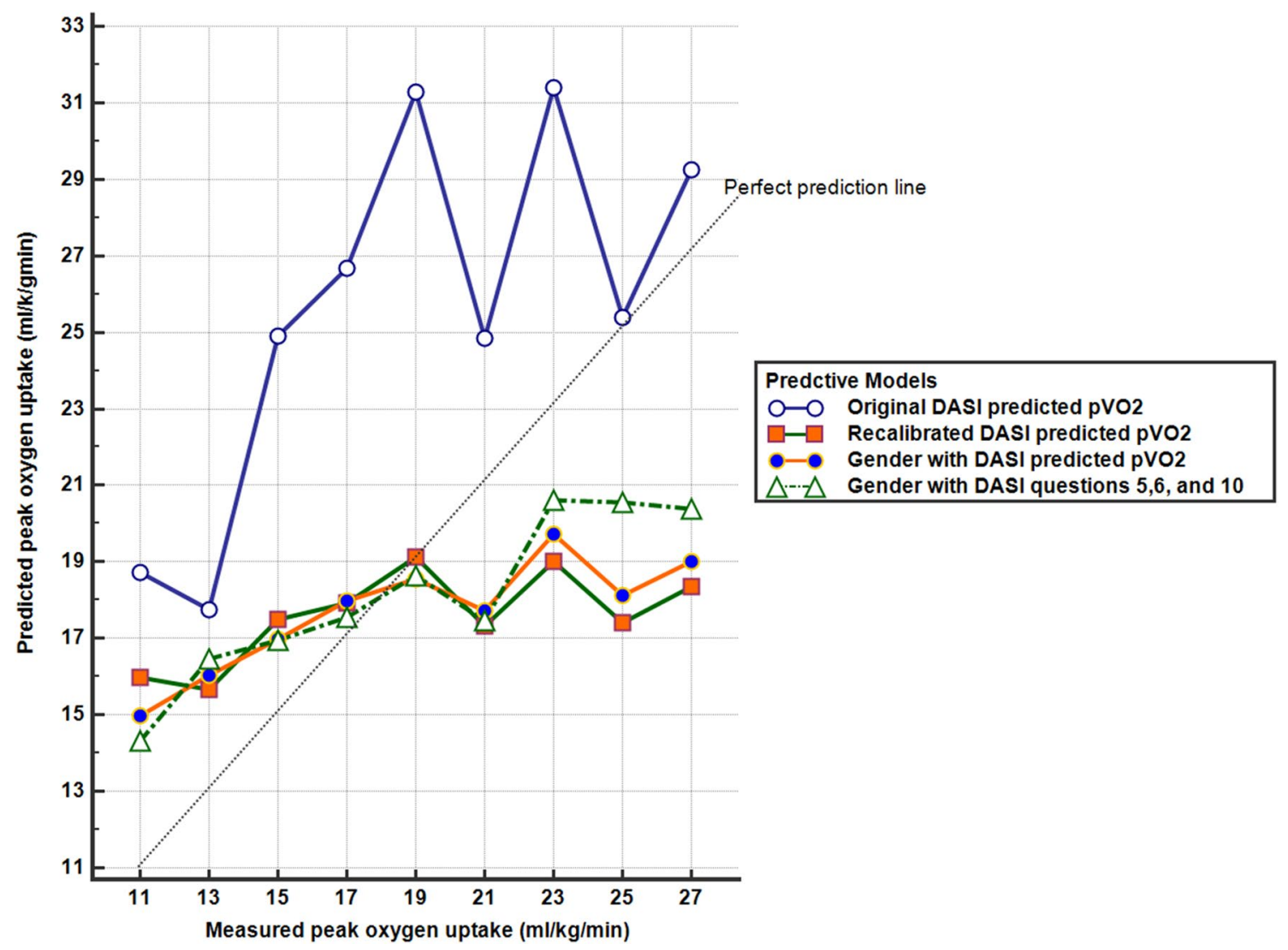

Fig. 8 Relationships between different models predicted peak oxygen uptake and measured oxygen uptake

perioperative setting, particularly more so in oncological patients. Similarly, the DASI or our modified model of the DASI is still not sufficiently reliable as a triage tool to identify those with high likelihood of low functional capacity (AUROC $<0.55$ for AT $<11 \mathrm{ml} \mathrm{kg}^{-1} \mathrm{~min}^{-1}$ and AUROC $<0.80$ for $\mathrm{pVO}_{2}<15 \mathrm{ml} \mathrm{kg} \mathrm{km}^{-1}$ ) who should be referred to a CPET laboratory for objective confirmation of their functional capacity.

\section{Potential causes for a further reduction in capacity for prediction}

Differences between our results and that of the previous studies may be due to differing methods of data collection for health quality outcomes, compounded by different settings and patient cohorts $[22,23]$. The initial DASI study population $\left(R^{2}=0.64\right)$ was interviewer-administered which had a higher predictive ability, but this was not confirmed by a validation cohort $\left(R^{2}=0.31\right)$ when a redesigned selfadministered questionnaire was used. Our results were more consistent with the latter, possibly due to wide interindividual variations in interpreting the meaning of the questions or recall bias. In addition, the medical and social context of the patients could also have an influence on the survey results. In large observational studies of healthy populations, participants have a tendency to overpredict their physical activity [24], particularly at higher levels of exercise [25]. Of note, our results also showed that DASI-predicted $\mathrm{pVO}_{2}$ did tend to overpredict the actual $\mathrm{pVO}_{2}$ values, suggesting that this questionnaire is less reliable when predicting functional capacity at higher $\mathrm{pVO}_{2}$ ranges. Similar subjective measures of functional capacity can be found in clinicianelicited stair-climbing ability, which has been incorporated into common practice to estimate perioperative risk [26, 27]. While history taking provides a quick bedside assessment of functional capacity, such subjective measures make them unreliable when administered by different parties. Furthermore, substantial discrepancy is reported between clinicianassessment and self-reported questionnaires in assessing functional capacity [15].

The tendency for the DASI, originally developed for use in the general population, to overpredict peak $\mathrm{VO}_{2}$ in major cancer surgical patients may also be related to the underlying pathophysiology of cancer and associated therapy, with patients likely to overestimate their functional capacity using the DASI questionnaire, because they are likely to recall what they could do prior to their cancer diagnosis. Over the course of 1-2 months, neoadjuvant chemotherapy 
rapidly decreases $\mathrm{pVO}_{2}$ by as much as $3 \mathrm{ml} \mathrm{kg}^{-1} \min ^{-1}[2$, 4]. Such rapid changes may make it difficult for patients to accurately estimate their current level of physical activity compared to that prior to commencement of neoadjuvant chemoradiotherapy. Recent studies have shown that exercise prescription during neoadjuvant therapy offset this decline in functional capacity [28]. This recall bias and acute decline in functional capacity may, indeed, be an inherent limitation for any form of subjective assessment of functional capacity in major cancer surgical patients, especially the elderly that have become deconditioned due to lifestyle choice or illness. It is worth noting that, at lower $\mathrm{pVO}_{2}$, the difference between the predicted and measured $\mathrm{pVO}_{2}$ values was smaller, and it may be that patients with extremely poor functional capacity may be accurately predicted by DASI.

In the original study by Hlatky et al. [14], subjects in the development phase had high $\mathrm{pVO}_{2}$ in excess of $30 \mathrm{ml} \mathrm{kg}-1$ $\min ^{-1}$ with correspondingly high DASI scores, whereas this was less so in the second validation cohort. In the population studied by Struthers et al.[16], 78\% of patients compared to our study's $67 \%$ of patients had a $\mathrm{pVO}_{2}>15 \mathrm{ml} \mathrm{kg}^{-1}$ $\min ^{-1}$. Therefore, it is likely that the DASI is more suited at predicting a high $\mathrm{pVO}_{2}$ in the fitter, general population, compared to the oncological population, who are more limited in functional capacity.

This study has some limitations. First, this was a singlecentre study: our results may not be applicable to patients with very different age and co-existing diseases to our patients. Second, due to the retrospective nature of this study, we did not have the opportunity to confirm whether our patients' answers to the DASI questionnaires were accurate by cross-checking the answers to the questionnaires with their next of kin. This study may have also been subjected to selection bias; although perioperative CPET referral guidelines are in place, surgical teams are responsible for CPET referrals, and in that, may have their own selection bias. Finally, the small sample size of this study limited the statistical power, precision of the estimates, and did not allow us to assess smaller sub-groups such as separating chemotherapy and radiotherapy, or whether DASI scores were noninferior in predicting perioperative morbidity and mortality compared to CPET measured $\mathrm{pVO}_{2}$.

Given the inverse relationship between functional capacity and postoperative complications [29, 30], developing a reliable tool to assess functional capacity (or to triage at-risk patients to resource-intensive CPET facilities for objective quantification of functional capacity) in surgical patients is essential to improve surgical outcomes, and especially to advance surgical oncology care. The DASI-predicted $\mathrm{pVO}_{2}$ had a weak relationship to objectively measure $\mathrm{pVO}_{2}$ with a large bias and wide range in limits of agreement in patients pending major cancer surgery. Both the total DASI scores and our permutations of the DASI-derived-predicted
$\mathrm{pVO}_{2}$ also could not be considered sufficiently accurate to replace actual CPET measured anaerobic threshold and $\mathrm{pVO}_{2}$. A high specificity (90\%) for a screening test (e.g., DASI) is needed to avoid missing too many patients with a low functional capacity before major cancer surgery. When the DASI's specificity was $90 \%$, the sensitivity would be $20 \%$ or less (Fig. 6). This would mean that using DASI as a screening test will, at best, reduce $20 \%$ of the CPET workload but still missing $10 \%$ of those with a low functional status (measured $\mathrm{PVO}_{2}<15 \mathrm{ml} \mathrm{kg}^{-1} \mathrm{~min}^{-1}$ ) compared to a strategy of not using DASI at all and performing only CPET for every major surgical cancer patient. Although the DASIpredicted $\mathrm{pVO}_{2}$ and measured $\mathrm{pVO}_{2}$ were not interchangeable or with large observed limits of agreement in general, the difference between the two parameters did appear to vary dependent on the observed value of $\mathrm{pVO}_{2}$ especially at low values of $\mathrm{pVO}_{2}$. As such, further studies evaluating DASI for those with a genuinely low $\mathrm{pVO}_{2}$ due to limited cardiopulmonary exercise capacity are warranted.

Acknowledgements We would like to acknowledge Ms. Yessim Karabiyik for her role in this project in data collection and administering the Duke Activity Status Index. Dr Ho would like to thank Raine Medical Research Foundation and WA Health for their funding through the Raine Clinical Research Fellowship.

\section{Compliance with ethical standards}

Conflict of interest Dr Ho is funded by WA Health and Raine Medical Research Foundation through the Raine Clinical Research Fellowship. The funding agencies have no influence on the choice of the subject matter, design of the study, data analyses, the decision to publish the results and the final content of the manuscript. The other authors have not received any funding and have no conflicts of interest to declare.

\section{References}

1. Silver JK, Baima J. Cancer prehabilitation: an opportunity to decrease treatment-related morbidity, increase cancer treatment options, and improve physical and psychological health outcomes. Am J Phys Med Rehabil. 2013;92(8):715-27.

2. West MA, Loughney L, Barben CP, Sripadam R, Kemp GJ, Grocott MP, Jack S. The effects of neoadjuvant chemoradiotherapy on physical fitness and morbidity in rectal cancer surgery patients. Eur J Surg Oncol. 2014;40(11):1421-8.

3. West MA, Loughney L, Lythgoe D, Barben CP, Adams VL, Bimson WE, Grocott MP, Jack S, Kemp GJ. The effect of neoadjuvant chemoradiotherapy on whole-body physical fitness and skeletal muscle mitochondrial oxidative phosphorylation in vivo in locally advanced rectal cancer patients-an observational pilot study. PLoS One. 2014;9(12):e111526.

4. Jack S, West MA, Raw D, Marwood S, Ambler G, Cope TM, Shrotri M, Sturgess RP, Calverley PM, Ottensmeier CH, Grocott MP. The effect of neoadjuvant chemotherapy on physical fitness and survival in patients undergoing oesophagogastric cancer surgery. Eur J Surg Oncol. 2014;40(10):1313-20.

5. Guazzi M, Adams V, Conraads V, Halle M, Mezzani A, Vanhees L, Arena R, Fletcher GF, Forman DE, Kitzman DW, Lavie CJ, 
Myers J. Clinical recommendations for cardiopulmonary exercise testing data assessment in specific patient populations. Eur Heart J. 2012;33(23):2917.

6. Older P, Hall A, Hader R. Cardiopulmonary exercise testing as a screening test for perioperative management of major surgery in the elderly. Chest. 1999;116(2):355-62.

7. Older P, Smith R. Experience with the preoperative invasive measurement of haemodynamic, respiratory and renal function in 100 elderly patients scheduled for major abdominal surgery. Anaesth Intensive Care. 1988;16(4):389-95.

8. Levett DZ, Grocott MP. Cardiopulmonary exercise testing, prehabilitation, and enhanced recovery after surgery (ERAS). Can J Anaesth. 2015;62(2):131-42.

9. Levett DZ, Grocott MP. Cardiopulmonary exercise testing for risk prediction in major abdominal surgery. Anesthesiol Clin. 2015;33(1):1-16.

10. Moran J, Wilson F, Guinan E, McCormick P, Hussey J, Moriarty $\mathrm{J}$. Role of cardiopulmonary exercise testing as a risk-assessment method in patients undergoing intra-abdominal surgery: a systematic review. Br J Anaesth. 2016;116(2):177-91.

11. Smith TB, Stonell C, Purkayastha S, Paraskevas P. Cardiopulmonary exercise testing as a risk assessment method in non cardio-pulmonary surgery: a systematic review. Anaesthesia. 2009;64(8):883-93.

12. Jones LW, Liang Y, Pituskin EN, Battaglini CL, Scott JM, Hornsby WE, Haykowsky M. Effect of exercise training on peak oxygen consumption in patients with cancer: a meta-analysis. Oncologist. 2011;16(1):112-20.

13. Chatterjee S, Sengupta S, Nag M, Kumar P, Goswami S, Rudra A. Cardiopulmonary exercise testing: a review of techniques and applications. J Anesth Clin Res. 2013;4(7):1-6.

14. Hlatky MA, Boineau RE, Higginbotham MB, Lee KL, Mark DB, Califf RM, Cobb FR, Pryor DB. A brief self-administered questionnaire to determine functional capacity (the Duke Activity Status Index). Am J Cardiol. 1989;64(10):651-4.

15. Stokes JW, Wanderer JP, McEvoy MD. Significant discrepancies exist between clinician assessment and patient self-assessment of functional capacity by validated scoring tools during preoperative evaluation. Perioper Med (Lond). 2016;5:18.

16. Struthers R, Erasmus P, Holmes K, Warman P, Collingwood A, Sneyd JR. Assessing fitness for surgery: a comparison of questionnaire, incremental shuttle walk, and cardiopulmonary exercise testing in general surgical patients. Br J Anaesth. 2008;101(6):774-80.

17. ATS/ACCP Statement on cardiopulmonary exercise testing. Am J Respir Crit Care Med. 2003;167(2):211-77.
18. Beaver WL, Wasserman K, Whipp BJ. A new method for detecting anaerobic threshold by gas exchange. J Appl Physiol. 1986;60(6):2020-7.

19. Pencina Michael J, Fine Jason P, D’Agostino Ralph B. Discrimination slope and integrated discrimination improvementproperties, relationships and impact of calibration. Stat Med. 2016;36(28):4482-90.

20. Vickers RR. Measurement error in maximal oxygen uptake tests. San Diego: Naval Health Research Center; 2003. Contract No.: 04-03.

21. Yap BW, Sim CH. Comparisons of various types of normality tests. J Stat Comput Simul. 2011;81(12):2141-55.

22. Anderson JP, Bush JW, Berry CC. Classifying function for health outcome and quality-of-life evaluation. Self-versus interviewer modes. Med Care. 1986;24(5):454-69.

23. Picavet HS, van den Bos GA. Comparing survey data on functional disability: the impact of some methodological differences. J Epidemiol Community Health. 1996;50(1):86-93.

24. Troiano RP, Berrigan D, Dodd KW, Masse LC, Tilert T, McDowell M. Physical activity in the United States measured by accelerometer. Med Sci Sports Exerc. 2008;40(1):181-8.

25. Ainsworth BE, Bassett DR Jr, Strath SJ, Swartz AM, O'Brien WL, Thompson RW, Jones DA, Macera CA, Kimsey CD. Comparison of three methods for measuring the time spent in physical activity. Med Sci Sports Exerc. 2000;32(9 Suppl):S457-64.

26. Reilly DF, McNeely MJ, Doerner D, et al. Self-reported exercise tolerance and the risk of serious perioperative complications. Arch Intern Med. 1999;159(18):2185-92.

27. Boult M, Howell S, Cowled P, De Loryn T, Fitridge R. Selfreported fitness of American Society of Anesthesiologists class 3 patients undergoing endovascular aneurysm repair predicts patient survival. J Vasc Surg.62(2):299-303.

28. West MA, Loughney L, Lythgoe D, Barben CP, Sripadam R, Kemp GJ, Grocott MP, Jack S. Effect of prehabilitation on objectively measured physical fitness after neoadjuvant treatment in preoperative rectal cancer patients: a blinded interventional pilot study. Br J Anaesth. 2015;114(2):244-51.

29. West MA, Parry MG, Lythgoe D, Barben CP, Kemp GJ, Grocott MP, Jack S. Cardiopulmonary exercise testing for the prediction of morbidity risk after rectal cancer surgery. Br J Surg. 2014;101(9):1166-72.

30. Nagamatsu Y, Shima I, Yamana H, Fujita H, Shirouzu K, Ishitake T. Preoperative evaluation of cardiopulmonary reserve with the use of expired gas analysis during exercise testing in patients with squamous cell carcinoma of the thoracic esophagus. J Thorac cardiovasc Surg. 2001;121(6):1064-8. 